\title{
Opportunistic Secure Communications for Wireless Local Area Networks
}

\author{
Il-Gu Lee \\ Department of Convergence Security Engineering, Sungshin University \\ iglee@sungshin.ac.kr
}

\begin{abstract}
In wireless local area networks (WLANs), if the channel medium is busy due to long reservation time by a station's medium access, other stations must wait for the medium busy time, interframe spaces and a random backoff period. This contention-based medium-access mechanism provides collision avoidance, but it limits wireless system performance; thus, service quality and throughput efficiency are severely degraded in highly dense networks. In this paper, an interference-aware opportunistic secure communications (OSC) scheme is proposed. The OSC scheme allows the wireless stations to transmit their data information to a neighbor node which has a high link quality in an interference perspective through an idle channel. In next contention, the neighbor node has a higher priority to transmit the aggregated data frames, which include data information from other stations to an access point (AP). The evaluation results confirm that the OSC can improve network throughput and station throughput by more than 5 times in dense network conditions, compared with the conventional channel-access mechanism.
\end{abstract}

Keywords: Interference-aware, medium-access control, CSMA/CA, opportunistic transmission, dense network, WLAN.

\section{Introduction}

Wireless local area networks (WLANs) are rapidly becoming pervasive, connecting massive devices worldwide in highly dense networks to offer wireless connectivity for seamless and high-quality services [1-3]. WLAN standards have evolved immensely over the years based on carrier-sense multiple access with collision avoidance (CSMA/CA), and they provide backward compatibility with previous versions [4]. In WLANs, each station signals its intent to transmit data frames before doing so. The CSMA/CA channelaccess mechanism adopts a random backoff algorithm that determines wait time before trying to use the channel. When the backoff time expires, the station check the medium status again to verify a clear channel. If the channel is still busy, another backoff interval is initiated that is shorter than the previous one. The process continues until that wait time reaches zero, and the channel is clear. Since this "listen before taking" method is used in CSMA/CA networks, any station wanting to transmit data must first verify that the channel is clear before transmitting, thereby avoiding potential collisions [5-7].

In a typical WLAN, stations connected to one network access point (AP) contend with each other to acquire a transmission opportunity (TXOP) for communicating with the AP. Furthermore, 802.11-based WLANs allow a station to acquire a long TXOP for a predetermined period in order to improve throughput efficiency by reducing communication overheads [8]. During the period in which a station having acquired a TXOP occupies a given channel, the other stations wait in a standby mode until the transmission period is terminated. That is, due to the protocol characteristic of the

Received (September 7, 2017), Review Result (December 1, 2017), Accepted (December 7, 2017) 
CSMA/CA, only a specific station is allowed to communicate with the AP by using a given channel resource during a specific time, based on the competition and avoidance scheme using a back-off algorithm and a carrier-sense algorithm.

However, as more wireless Internet of Things are connected in the limited channel frequency, the failure probability of packet communications is expected to increase due to intentional and unintentional interference from other devices $[2,3,9]$. In dense networks, the conventional channel access mechanism is not effective in terms of channel utilization efficiency when multiple basic service sets (BSSs) are overlapped in dense networks. The factors contributing to the inefficiency can be summarized as follows.

First, the transmission and reception performance of the stations and the environments around the stations vary. However, WLAN technology adopts a method in which the stations contend with each other to acquire a TXOP on a shared medium according to the CSMA/CA protocol, without considering these differences. A station acquiring a TXOP is determined on the basis of a simple backoff scheme, without reflecting the link performance with the AP during the TXOP acquisition process. Therefore, when a station having poor link performance with the AP occupies a channel, it may cause a reduction in the general performance of the network.

Second, it is not common that all the channels of the ISM bands are always used in the real world. However, when an AP and one station communicate with each other through the CSMA/CA protocol, all stations except for one station should be in a standby state until the TXOP period of the terminal occupying a channel is terminated. Therefore, when a specific station occupies a channel, the remaining channels are not used.

Third, as the number of stations in a BSS increases, the throughput efficiency of the conventional competition and collision avoidance scheme decreases due to overheads caused by the backoff time, interframe space (IFS), and packet header. When a relatively short packet is transmitted during the TXOP period acquired through the contention among multiple stations and the channel occupation is terminated, the efficiency is further degraded.

Channel interference effect and protection mechanisms have been investigated. However, their scope has been confined to analyzing interferences effects on legacy $11 \mathrm{a} / \mathrm{b} / \mathrm{g} / \mathrm{n}$ wireless LAN systems $[10,11]$, while other studies have suggested using centralized channel assignment techniques or channel hopping [12, 13]. However, the channel hopping schemes are not effective due to protocol overheads. Also, the protection mechanisms of [14-16] require modification of the current standard. In [17, 18], the authors proposed interference-aware self-optimizing receiver design, but the solutions are limited in their ability to mitigate the interference effect on the receiver.

In this paper, an interference-aware opportunistic secure communications (OSC) scheme is proposed. The OSC scheme allows the waiting stations to transmit their data frames to a relaying node which has a high link quality through an idle channel. In the next contention after the prior TXOP, the relaying node has a higher priority to transmit the aggregated frames to the AP after receiving data frames from other stations. The evaluation results demonstrate the significantly improved performance of the OSC scheme compared with the conventional channel access schemes in dense networks. This work is the first work to improve network throughput in dense networks for CSMA/CAbased WLANs allowing simultaneous transmissions through a secondary channel by stations waiting for the primary channel during TXOP. The proposed OSC scheme overcomes the disadvantage of the existing CSMA/CA protocol that it does not consider differential channel environments among the AP and the stations by allowing stations to optimize the network before taking next TXOP.

The remainder of this paper is organized as follows. In Section 2, the conventional channel access mechanism is overviewed. In Section 3, the proposed interference-aware transmission opportunity scheme is proposed. In Section 4, the evaluation results are presented, and finally Section 5 draws conclusions. 


\section{Conventional Channel Access Mechanism}

\subsection{Protocol Overheads}

Traditionally, WLAN technologies have been evolving to improve throughput by reducing communication overheads, and improving operation efficiency. Therefore, throughput is an important performance metric in the design and optimization of WLANs. Throughput can be obtained by dividing a successfully transmitted payload length by the total transmission time. Throughput differs depending on channel states. When the channel state is poor, packet errors frequently occur, and the number of retransmissions increases. Then, the throughput efficiency is degraded as much by overheads caused by retransmission time including the additional transmission time, the IFS time, and the backoff time.

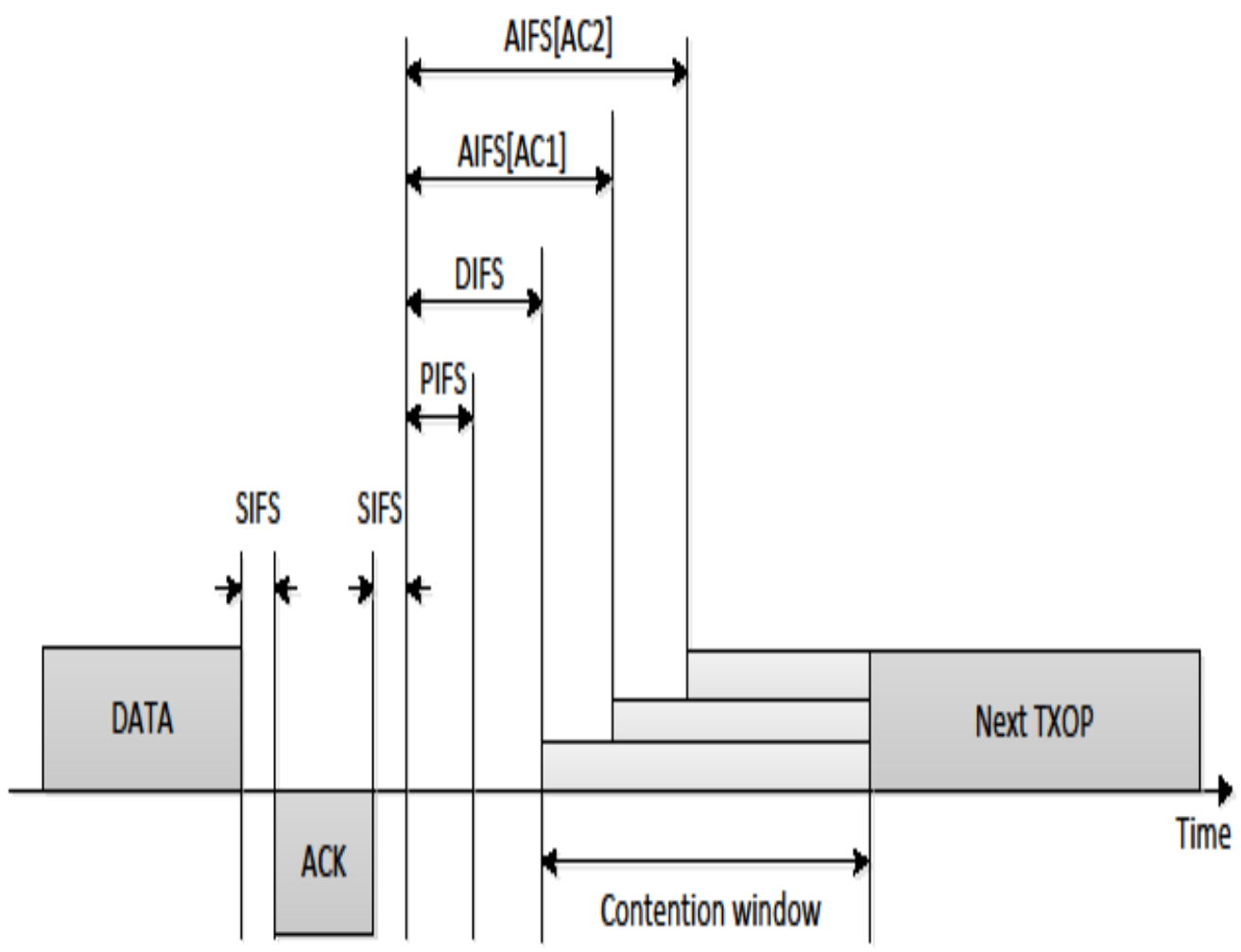

Figure 1. Packet Transmission Protocol and Overhead in WLAN

Figure 1 is a diagram showing a packet transmission protocol of the WLAN standard and the overhead according to the packet transmission protocol. When a data frame is transmitted, an acknowledge (ACK) signal corresponding to the data is transmitted after a short interframe space (SIFS), which is a predetermined short interframe time value. Even when an ACK signal is transmitted, a time corresponding to an SIFS, which is the minimum value for a subsequent transmission, needs to pass. After that, transmission is performed after a point coordination function (PCF) interframe space (PIFS), a distributed coordination function (DCF) interframe space (DIFS), an arbitration interframe space (AIFS[AC1]), or an AIFS [AC2]. The time after the DIFS, the AIFS[AC1], or the AIFS[AC2] includes a contention window. As described above, the interframe spaces, ACK transmission time, and contention windows occurring after the transmission of the respective data serve as overheads that degrade the throughput efficiency of data transmission. 


\subsection{Communication Overheads}

In addition, when an AP handles multiple stations, the channel states between the AP and the stations may differ depending on the positions of the stations and the surrounding environments, such as interference caused by other wireless equipment, obstacles, and refraction and reflection of waves. In the WLAN standard, however, stations may acquire TXOPs according to their own backoff algorithm without considering the differential channel environments among the AP and the stations.

Figure 2 is a timing diagram showing channel access among an AP and five stations, in which a TXOP is acquired according to the WLAN technology. While STA1 occupies a channel from time T1 to T2, that is, during the TXOP period of STA1, STA2 to STA5 should wait until the channel occupation of STA1 is terminated. Even though an uplink traffic case is shown in this figure, a downlink traffic case would be the same. Even when one station occupies the channel after time T2, the other stations should be in a standby state. Regardless of the channel states, all the stations contend with each other to acquire a TXOP and then transmit data. In short, the WLAN technology that is currently used is not effective in terms of network throughput and channel utilization.

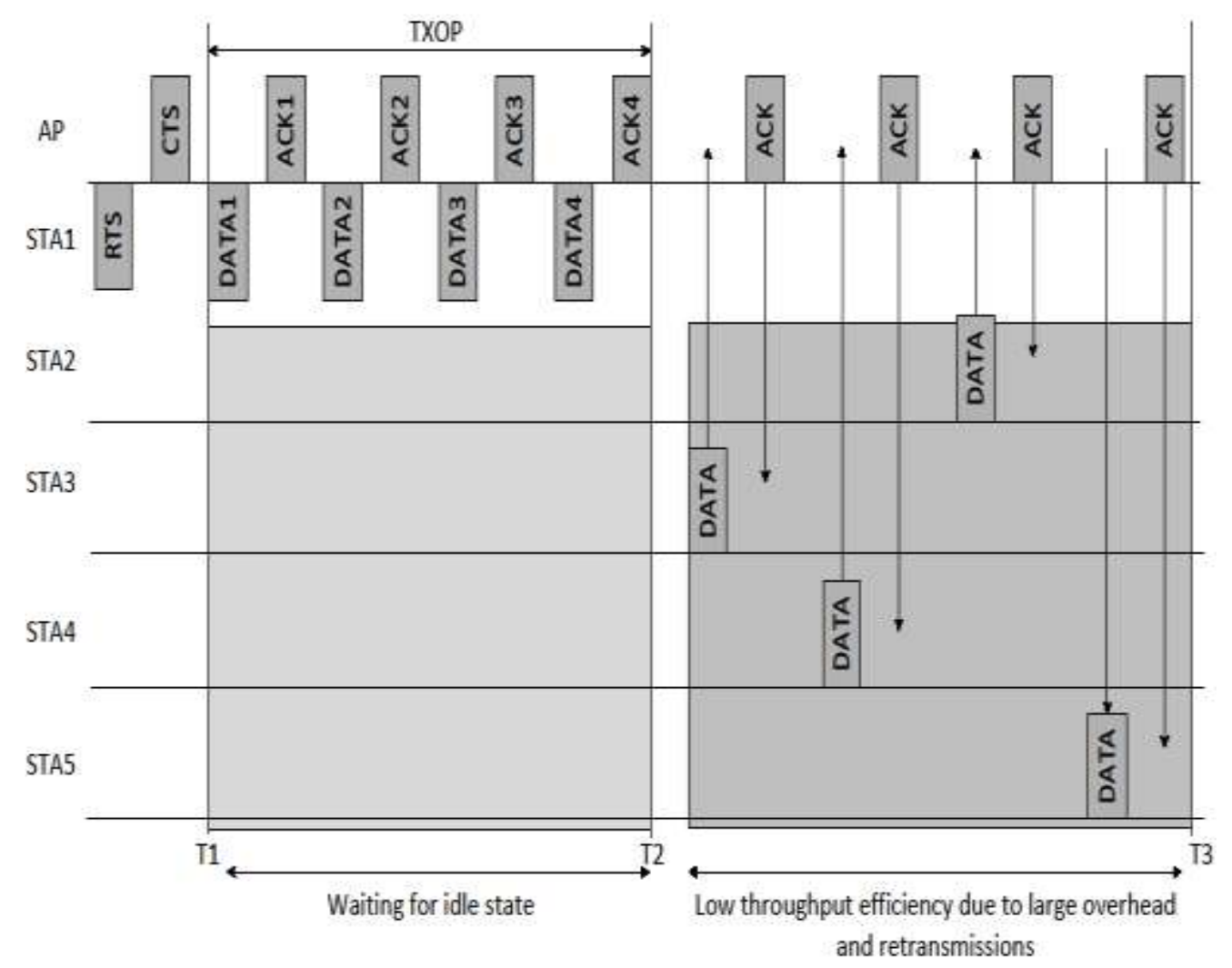

Figure 2. TXOP Operation in CSMA/CA

\section{Interference-Aware Opportunistic Transmission}

In this section, an interference-aware opportunistic secure communications (OSC) method is proposed to improve the throughput performance of WLANs in dense networks in the order of its operation, methodology, and analytical model.

\subsection{OSC Operation}

In the proposed OSC approach, while a specific station transmits data in a channel for which a TXOP is acquired through the contention-based CSMA/CA protocol, other 
stations that did not acquire a TXOP transmit their data to a station having a favorable channel state with the AP by using an adjacent channel that is not being used at that time. After that, when the channel occupation of the specific station is terminated, the station having the favorable channel state acquires a channel occupation priority.

While a specific station communicates with the AP through a specific channel in a BSS, the other stations perform communication to improve the frame delivery ratio and throughput efficiency by using one of the remaining frequency channels. That is, when a specific station communicates with the AP in the BSS, the network is set in an optimal state to effectively use the channel in the next TXOP. Furthermore, short packets transmitted by multiple stations through the remaining channels are aggregated and then transmitted to the AP through a channel having a high quality. Using such a method, it is possible to improve the overall throughput efficiency and the channel utilization.

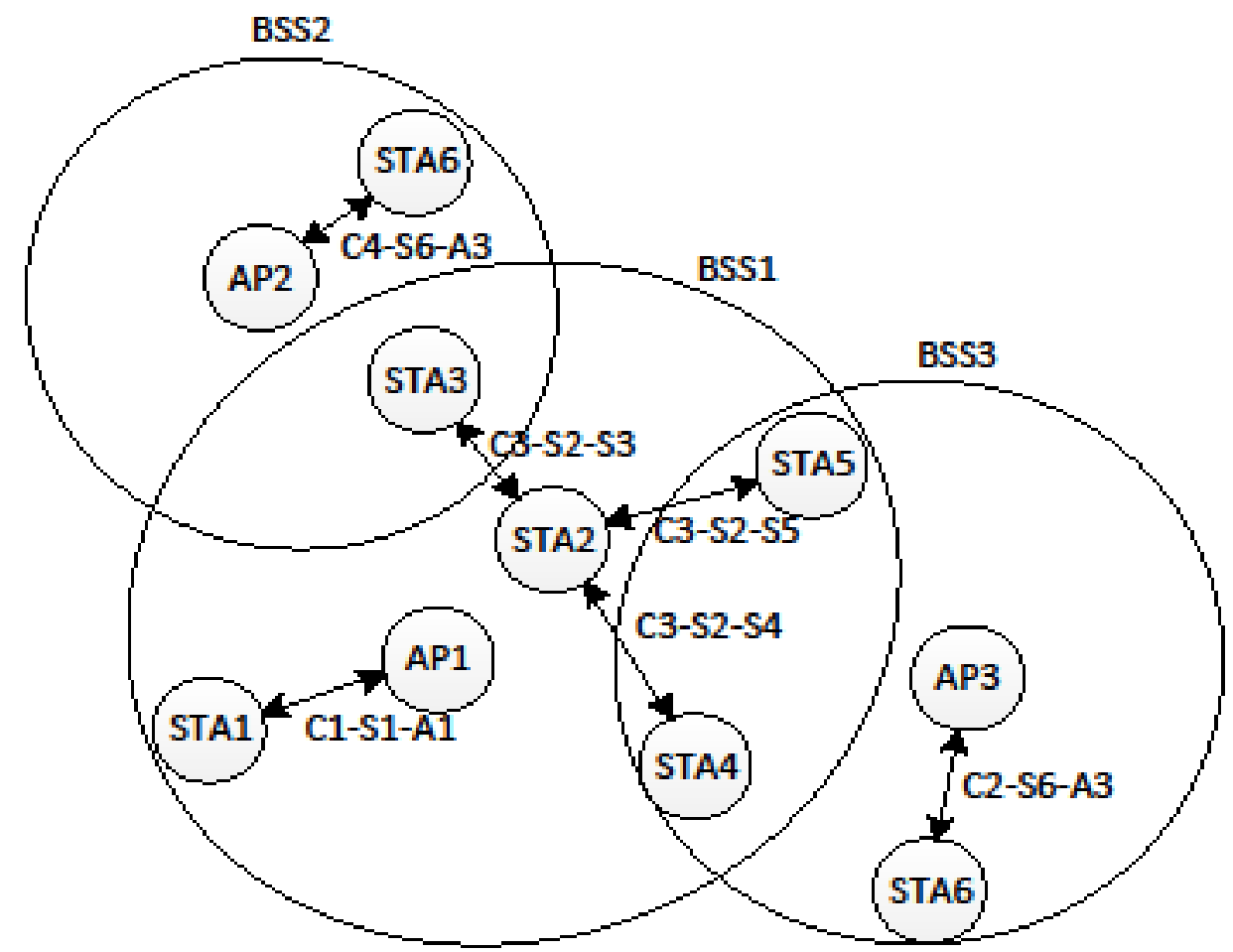

Figure 3. OSC Operation in Dense Network Condition

Figure 3 is a diagram showing a network configuration for OSC operation in a dense network condition. In this figure, basic service sets (BSS) determined by the respective APs AP1 to AP3 are discriminated and represented by BSS1, 2, and 3. Furthermore, the communications between the respective stations and the APs or the communications between the stations are indicated by two-way arrows. The communications may be described as follows. Here, CX-SY-AZ on a two-way arrow represents an example in which station Y communicates with AP Z through channel X. For example, C1-S1-A1 means that STA1 (S1) communicates with AP1 (A1) through channel 1 (C1).

When the STA1 occupies channel 1 according to the existing CSMA/CA protocol, stations STA3, STA4, and STA5 transmit data to STA2, which statistically has the most favorable channel state with AP1, by using channel 3 which is not used while STA1 uses channel 1. When the channel occupation of STA1 is terminated, a long TXOP corresponding to the length of packets to be transmitted by STA3, STA4, and STA5 is preferentially given to STA2.

Packets that were scheduled to be transmitted by multiple stations are aggregated to minimize the protocol and communication overheads through a station that has a good 
link quality with AP. Therefore, the retransmission rate is reduced to improve the overall throughput. The backoff time for channel occupation contention, the IFS, and the packet header overhead are reduced. Accordingly, the throughput efficiency and channel resource utilization are improved. Therefore, the BSS1 of AP1 utilizes two channels, C1 and C3, at the same time. That is, when STA2 acquires a TXOP to occupy channel C1, the terminals STA1, STA3, and STA4 use channel C3 to relay data to the terminal STA2, which is of the greatest advantage in acquiring the next TXOP.

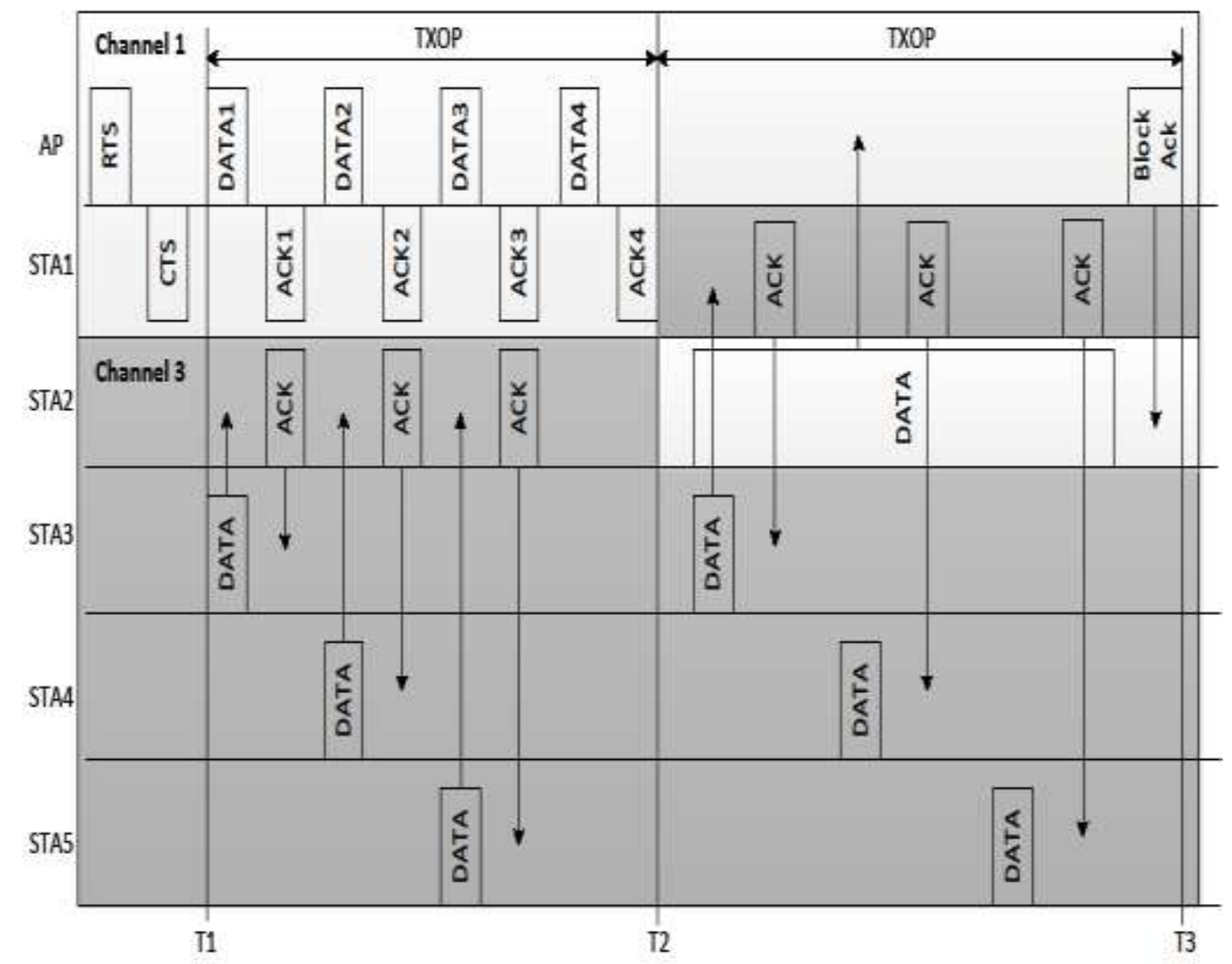

Figure 4. OSC Operation in Dense Network

Figure 4 shows a timing diagram in which data frames are transmitted in OSC operation. In this figure, first communication from $\mathrm{T} 1$ to $\mathrm{T} 2$ is performed between the STA1 and the AP through channel 1. In the conventional method, even when one station occupies only channel 1 , the other stations should wait before they acquire the channel through a subsequent contention period. However, simultaneous communications among the other terminals can be performed in the proposed OSC scheme. At this time, the communications include a case in which a station transmits data to another station, STA2, which is of great advantage in communicating with the AP, such that the data is relayed.

After data frames are transmitted to STA2 from other stations, a channel between the terminal STA2 and the AP is formed through a contention with higher priority in the time from T2 to T3, and STA2 transmits the data frame to the AP through a selected channel, for example, channel 3. At this time, STA2 aggregates the data frames received from other stations and then transmits the aggregated data. Furthermore, the AP informs STA2 of the result of the data frames received from STA2 through a block ACK (BA) signal.

The AP stores the statistical link quality of the stations communicating with the AP and state information. Then, the AP informs stations belonging to the BSS of multiple stations that are of advantage in acquiring a subsequent TXOP through a control signal. The AP also periodically senses idle channels and builds an available channel list utilizing 
spectrum-sensing schemes $[19,20]$ and secondary antenna to scan the channels [21]. Thus, the AP can inform the stations belonging to the BSS of a channel which is currently used and a channel that is not used among the adjacent channels by using a control signal. Stations that do not occupy a channel contend with each other to acquire a TXOP in the remaining channels. When the channel information such as signal-to-interference-noise ratio (SINR), a packet error rate (PER) and the available channel list between AP and stations is shared, data frames are transmitted to a station having a favorable channel state with the AP in the order of terminals occupying the channels. After the channel occupation is terminated, priority is given to a station to which data packets are relayed and buffered when the next TXOP is determined.

The proposed method employs the OSC scheme for enhanced CSMA/CA. When a specific station occupies a channel for a long time, stations that did not occupy the channel transmit data to the station that has the best transmission efficiency when acquiring the next TXOP, for example, a station having a good link quality with the AP to be transmitted at the next time, or an arbitrary station, by using an idle channel that is not used. The station receiving the data preferentially acquires the next TXOP. Therefore, it is possible to improve the overall throughput and frequency efficiency. When the proposed method is applied, it is possible to increase the throughput efficiency, channel utilization, and network efficiency in wireless communication systems in which the stations acquire a TXOP according to the CSMA/CA protocol.

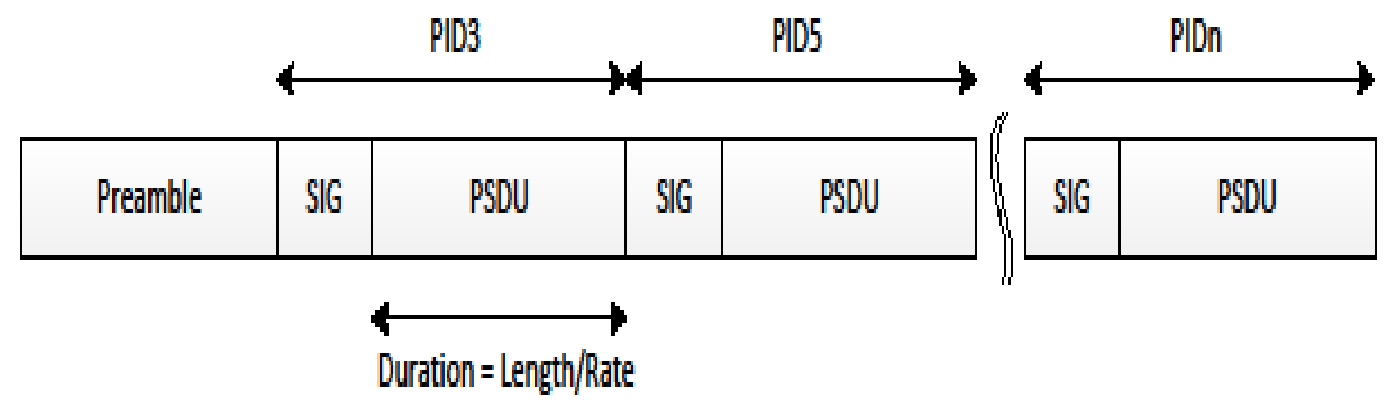

Figure 5. Aggregate PSDU Frame Format for OSC

Figure 5 shows an aggregated frame structure for the proposed OSC scheme. In the frame format, a single training sequence at the front of the physical layer convergence protocol (PLCP) protocol data unit (PPDU) is followed by a framing structure that consists of the physical layer (PHY) signaling fields delimiting PLCP service data units (PSDUs). The PHY signaling fields include length, rate, and PHY ID (PID) information. The length and rate information are used to inform the duration of the PSDU that follows. The PID indicates the source device's address of the PSDU. A bit in the signal field is used to indicate the last PSDUs in the aggregate. Therefore, data aggregated to a single station is more efficiently handled. The PID is encoded by BSS color bits to indicate the BSS index when normal frame transmission mode is applied while PIDs in multiple signal fields are partial association IDs for the destination stations' IDs.

Transmission over a wireless medium is error prone. Therefore, if a station receives a data frame addressed to it, it sends an ACK frame immediately. In the proposed OSC scheme, since data frames of multiple stations are delivered in the form of aggregated PSDUs through a relaying node, an ACK frame indicates the PID number as well as the PSDU sequence number in the form of block ACK. For example, STA1 is transferring data to the AP. STA3, 4, and 5 transmit data in an adjacent idle channel to STA2, which is selected as a relaying node. When the TXOP of STA1 is terminated and the medium is idle, STA2 accesses the medium and transmits the aggregated data frame to the AP. If the AP finishes demodulating the frame, it responds with a block ACK that includes a bitmap. 
The block ACK bitmap indicates the receive status of PSDUs corresponding to PIDs. Upon receiving the block ACK frame, the multiple originators discard acknowledged data frames and requeue the PSDUs not acknowledged. Then, each station begins channel access again to retransmit.

The AP allocates the relaying node and the secondary channel for simultaneous transmission so that all stations in the BSS contend and transmit to the relaying node through the secondary channel during the TXOP of STA1. The AP indicates that the relaying node's PID and the secondary channel index in the field of service field in RTS and CTS frames. This information can be delivered by newly defined request and response frames for OSC, but RTS and CTS frames can be used like the dynamic bandwidth allocation mechanism of IEEE 802.11ac standard [5-7].

\section{Performance Evaluation}

In this section, the evaluation methodology and results are presented. The proposed OSC scheme was evaluated using a computer simulation. To verify the functionality and performance of the OSC scheme, the simulation model was built on a bit- and cycleaccurate $\mathrm{C}$ simulator, which is the same model used for the implemented commercial chipset, and it was certified through standard Wi-Fi certification procedures [17].

Figure 6 presents the effect of error probability on throughput per station. In this experiment, 1,000 bytes payload length was assumed. As a result, a transmission attempt could fail due to channel errors caused by noise and interference. Interference and collision due to contending stations lowers the throughput per station. However, if the proposed OSC scheme is applied, the throughput is improved significantly. For example, the OSC scheme achieved a throughput of $25 \mathrm{Mbps}(10$ times higher) at 0.5 error probability, compared with $2.5 \mathrm{Mbps}$ when the number of stations was 5 . This is because the OSC scheme allows waiting stations to transmit data frames to a relay node that has a high-quality link to be optimized for the next TXOP. Then, the relay node aggregates the frames and obtains a TXOP with a higher priority. This interference-aware opportunistic property can improve the network throughput and throughput per station significantly.

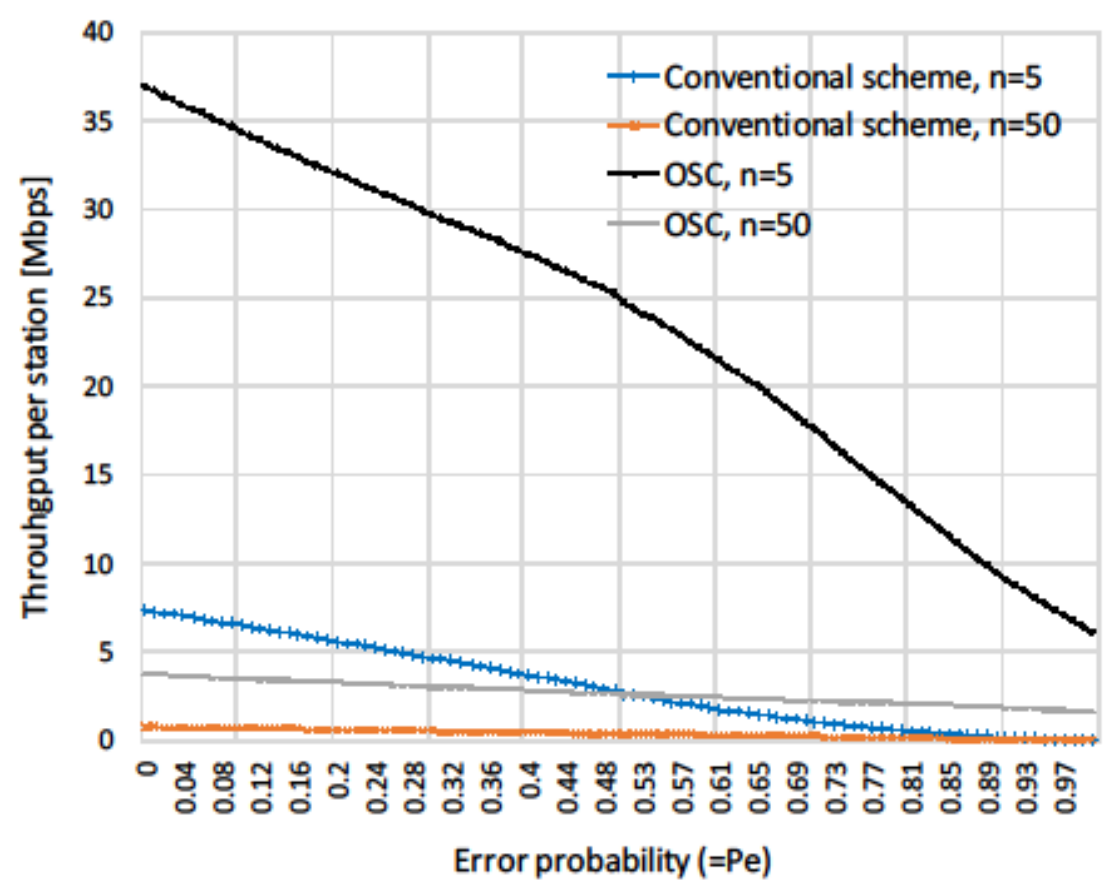

Figure 6. Throughput per Station vs. Error Probability 


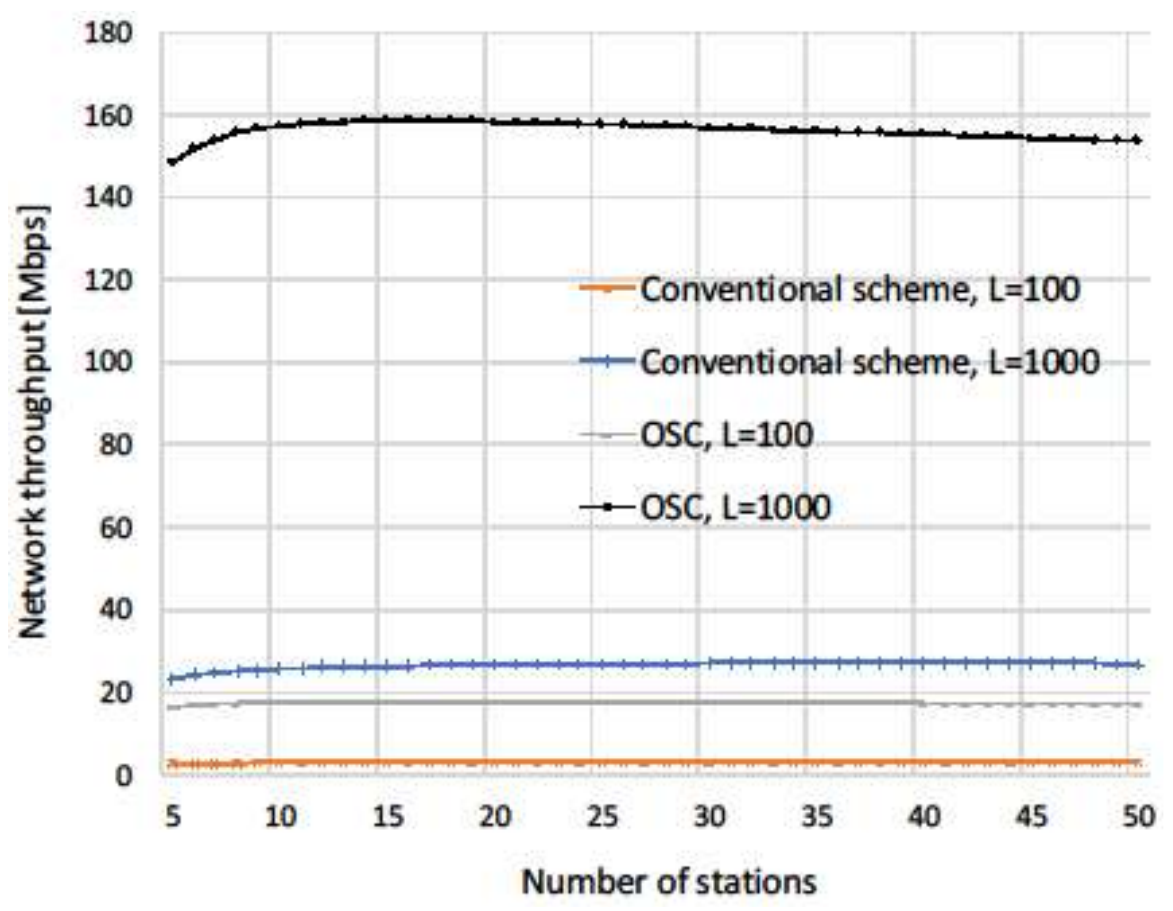

Figure 7. Network Throughput vs. Number of Stations

Figure 7 demonstrates the evaluation results for network throughput in relation to the number of stations in the BSS. In this experiment, PSDU lengths of 100 bytes or 1,000 bytes were assumed. The results clearly show the advantage of adopting the proposed OSC scheme. When the number of stations was increased, the throughput of the conventional transmission scheme was saturated in a very low network capacity range. If the proposed OSC scheme was applied, the network throughput was significantly improved. The overall throughput improvement of the OSC scheme was more than 6 times that of the conventional scheme.

\section{Conclusion}

In this paper, the limitations of the existing channel access mechanism in dense networks were investigated. Even though it is commonly implemented for Wi-Fi devices to communicate in the shared access to a wireless medium through carrier-sensing multiple access, the conventional medium-access mechanism makes wireless networks underutilized, coverage-limited, and capacity-limited.

To fully utilize network resources, the OSC scheme offers a means to improve network capacity and efficiency. Besides transmitting their own generated traffic to a good-quality relaying node, even if the channel of the BSS is reserved by a station, the relaying node is charged with forwarding traffic from stations routing through it. The proposed OSC scheme can mitigate the spectrum scarcity and low channel utilization caused by CSMA/CA in a static channel utilization. Recognizing that frequency resources are limited and not fully utilized, the proposed OSC approach is more useful because it improves channel utilization efficiency in dense networks. For future work, the proposed OSC approach will be implemented and demonstrated in a real testbed.

\section{Acknowledgements}

This work was supported by the Sungshin University Research Grant of 2017-1-28$002 / 1$. 


\section{References}

[1] Wi-Fi Alliance, "Connect your life: Wi-Fi and the Internet of Everything", Available online: http://www.wi-fi.org/system/files/wp_Wi-Fi_Internet_of_Things_Vision_20140110.pdf (accessed at May. 21, 2017).

[2] S. Tozlu, "Feasibility of Wi-Fi Enabled Sensors for Internet of Things", Wireless Communications and Mobile Computing Conference (IWCMC), 2011 7th International, (2011), pp. 291-296.

[3] S. Tozlu, M. Senel, W. Mao, A. Keshavarzian, "Wi-Fi enabled sensors for internet of things: A practical approach", IEEE Communications Magazine, vol. 50, (2012).

[4] W. Sun, O. Lee, Y. Shin, S. Kim, C. Yang, H. Kim, S. Choi, "Wi-Fi could be much more", IEEE Communications Magazine, vol. 52, (2014), pp. 22-29.

[5] IEEE Standard 802.11ac, IEEE Standard for Information technology - Telecommunications and information exchange between systems Local and metropolitan area networks - Specific requirements Part 11: Wireless LAN Medium Access Control (MAC) and Physical Layer (PHY) Specifications Amendment 4: Enhancements for Very High Throughput for Operation in Bands below $6 \mathrm{GHz},(\mathbf{2 0 1 3})$.

[6] IEEE Standard 802.11af, IEEE Standard for Information technology - Telecommunications and information exchange between systems - Local and metropolitan area networks - Specific requirements Part 11: Wireless LAN Medium Access Control (MAC) and Physical Layer (PHY) Specifications Amendment 5: Television White Spaces (TVWS) Operation, (2014).

[7] IEEE 802.11ah, IEEE Standard for Information Technology - Telecommunications and Information Exchange Between Systems - Local and Metropolitan Area Networks - Specific Requirements - Part 11: Wireless LAN Medium Access Control (MAC) and Physical Layer (PHY) Specifications: AmendmentSub $1 \mathrm{GHz}$ License-Exempt Operation, (2014).

[8] E. Perahia, R. Stacey, "Next generation wireless LANs: $802.11 \mathrm{n}$ and 802.11 ac", Cambridge university press, (2013).

[9] G. Goth, “Next-Generation Wi-Fi: As Fast as We'll Need?”, IEEE Internet Computing, vol. 16, pp.7-9, (2012).

[10] A. Zubow, R. Sombrutzki, “Adjacent Channel Interference in IEEE 802.11n”, Wireless Communications and Networking Conference (WCNC), (2012), pp.1163-1168.

[11] M. Doering, L. Budzisz, D. Willkomm, A. Wolisz, "About the practicality of using partially overlapping channels in IEEE 802.11 b/g networks", Communications (ICC), 2013 IEEE International Conference, (2013), pp.5110-5114.

[12] M. S. Gokturk, G. Ferazoglu, "Adjacent channel interference aware channel selection for wireless local area networks", Wireless Communications and Networking Conference (WCNC), (2014), pp.2922-2927.

[13] K. Zhou, X. Jia, L. Xie, Y. Chang, X. Tang, "Channel assignment for WLAN by considering overlapping channels in SINR interference model", International Conference on Computing, Networking and Communications (ICNC), (2012), pp.1005-1009.

[14] Arslan, M. Y., Pelechrinis, K., Broustis, I., Singh, S., Krishnamurthy, S. V., Addepalli, S., Papagiannaki, K., ACORN: An auto-configuration framework for 802.11 n WLANs, IEEE/ACM Transactions on Networking, vol. 21, (2013), pp. 896-909.

[15] S. Rayanchu, V. Shrivastava, S. Banerjee, R. Chandra, "FLUID: Improving Throughputs in Enterprise Wireless LANs through Flexible Channelization", IEEE Transactions on Mobile Computing, vol. 11, (2013), pp.1455-1469.

[16] T. Li, M. K. Han, A. Bhartia, L. Qiu, E. Rozner, Y. Zhang, B. Zarikoff, "CRMA: collision-resistant multiple access", Proceedings of the 17th annual international conference on Mobile computing and networking, pp.61-72, 2011.

[17] I. G. Lee, M. Kim, "Interference-aware self- optimizing Wi-Fi for high efficiency internet of things in dense networks", Computer Communications, vol. 89, (2016), pp.60-74.

[18] B. Kauffmann, F. Baccelli, A. Chaintreau, V. Mhatre, K. Papagiannaki, C. Diot, "Measurement-Based Self Organization of Interfering 802.11 Wireless Access Networks", INFOCOM 2007, 26th IEEE International Conference on Computer Communications, (2007), pp.1451-1459.

[19] E. Axell, G. Leus, E. G. Larsson, H. V. Poor, "Spectrum Sensing for Cognitive Radio: State-of-the-Art and Recent Advances", IEEE Signal Processing Magazine, (2012), pp.101-116.

[20] S. Shobana, R. Saravanan, R. Muthaiah, "Matched Filter Based Spectrum Sensing on Cognitive Radio for OFDM WLANs", International Journal of Engineering and Technology (IJET), vol. 5, (2013), pp.142.

[21] Cisco Meraki, MR34 datasheet. Available online: https://meraki.cisco.com/lib/pdf/meraki_datasheet_ MR34.pdf. (accessed at May. 21, 2017).

[22] G. Bianchi, "Performance analysis of the IEEE 802.11 distributed coordination function", IEEE Journal on selected areas in communications, (2000), pp.535-547.

[23] H. Zhai, Y. Kwon, Y. Fang, "Performance analysis of IEEE 802.11 MAC protocols in wireless LANs", Wireless communications and mobile computing, (2004), pp.917-931.

[24] Z. Hadzi-Velkov, B. Spasenovski, "Saturation throughput - delay analysis of IEEE 802.11 DCF in fading channel”, Communications, 2003. ICC'03. IEEE International Conference, vol. 1, (2003), pp.121126. 
[25] F. Daneshgaran, M. Laddomada, F. Mesiti, M. Mondin, "Unsaturated Throughput Analysis of IEEE 802.11 in Presence of Non Ideal Transmission Channel and Capture Effects", IEEE Transactions on Wireless Communications, Vol. 7, pp.1276-1286, 2008.

[26] Z. N. Kong, D. H. Tsang, B. Bensaou, D. Gao, "Performance analysis of IEEE 802.11e contention-based channel access", IEEE Journal on selected areas in communications, (2004), pp.2095-2106.

[27] L. Carious, et al., "High-efficiency WLAN, Standard document", IEEE 802.11-13/0331r5, (2013).

[28] E. Perahia, et al., "High-efficiency WLAN overview, Standard document", IEEE 802.11-14/0214r2, (2014).

\section{Author}

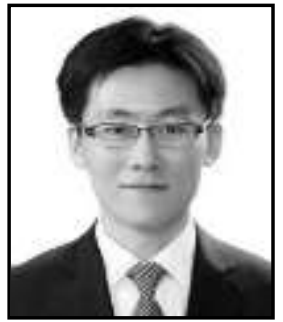

Il-Gu Lee, he is a professor at the Department of Convergence Security Engineering, Sungshin University (SU), Seoul, Korea. Before joining SU in March 2017, he was with the Electronics and Telecommunications Research Institute (ETRI) as a senior researcher from 2005 to 2017, and served as a principal architect and a project leader for Newratek (KR) and Newracom (US) from 2014 to 2017. $\mathrm{He}$ received his B.S. degree in electrical engineering from Sogang University, Seoul, Korea, at 2003, and his M.S. degree in the Department of Information and Communications Engineering from Korea Advanced Institute of Science and Technology (KAIST), Daejeon, Korea, at 2005. He also received his M.A degree in Intellecture Property from KAIST at 2012. He received his Ph.D degree in the Graduate School of Information Security in Computer Science \& Engineering Department from KAIST at 2016. His current research interests are in the area of wireless/mobile networks with an emphasis on information security, networks, wireless circuit and systems. He has authored/coauthored more than 34 technical papers in the areas of information security, wireless networks and communications, and holds about 132 patents. He is also an active participant of and contributor to the IEEE 802.11 WLAN standardization committee. 
International Journal of Control and Automation

Vol. 10, No. 12 (2017) 